\title{
The Expression of Adipophilin Is Frequently Found in Solid Subtype Adenocarcinoma and Is Associated with Adverse Outcomes in Lung Adenocarcinoma
}

\author{
Sun Ah Shin · Hee Young $\mathrm{Na}^{1}$ \\ Ji Young Choe ${ }^{2}$. Doohyun Chung \\ Mira Park ${ }^{3}$. Sohee $\mathrm{Oh}^{4}$ · Ji Eun Kim ${ }^{3}$ \\ Department of Pathology, Seoul National \\ University Hospital, Seoul; 'Department of \\ Pathology, Seoul National University Bundang \\ Hospital, Seongnam; '2Department of Pathology, \\ Hallym University Sacred Heart Hospital, Anyang; \\ Departments of ${ }^{3}$ Pathology and ${ }^{4}$ Biostatics, \\ Seoul National University Boramae Hospital \\ Seoul, Korea \\ Received: August 7, 2018 \\ Revised: September 5, 2018 \\ Accepted: September 13, 2018 \\ Corresponding Author \\ Ji Eun Kim, MD, PhD \\ Department of Pathology, Seoul National University \\ Boramae Hospital, 20 Boramae-ro 5-gil, \\ Dongjak-gu, Seoul 07061, Korea \\ Tel: $+82-2-870-2642$ \\ Fax: +82-2-831-026 \\ E-mail: npol181@snu.ac.kr
}

\begin{abstract}
Background: The up-regulation of the lipogenic pathway has been reported in many types of malignant tumors. However, its pathogenic role or clinical significance is not fully understood. The objective of this study was to examine the expression levels of adipophilin and related hypoxic signaling proteins and to determine their prognostic impacts and associations with the pathologic characteristics of lung adenocarcinoma. Methods: Expression levels of adipophilin, heat shock protein 27 (HSP27), carbonic anhydrase IX, and hypoxia-inducible factor $1 \alpha$ were examined by immunohistochemical staining using tissue microarray blocks. Correlations between protein expression levels and various clinicopathologic features were analyzed. Results: A total of 230 cases of primary adenocarcinoma of the lung were enrolled in this study. Adipophilin expression was more frequent in males and with the solid histologic type. It was correlated with HSP27 expression. Patients with adipophilin-positive adenocarcinoma showed a shorter progression-free survival (PFS) (median PFS, 17.2 months vs 18.4 months) in a univariable survival analysis, whereas HSP27 positivity correlated with favorable overall survival (OS) and PFS. In a multivariable analysis, adipophilin and HSP27 were independent prognostic markers of both OS and PFS. Conclusions: Activated lipid metabolism and the hypoxic signaling pathway might play a major role in the progression of lung adenocarcinoma, especially in the solid histologic type.
\end{abstract}

Key Words: Adipophilin; Lung adenocarcinoma; Hypoxia; Prognosis
Metabolic shifts, as well as mutations, are a distinguishing feature of cancer biology. ${ }^{1}$ Cancer cells can modify their metabolic pathways to obtain more energy required for proliferation or dissemination, initially affect glucose metabolism but largely in lipidcholesterol. Therefore, a high lipid content in cancer cells is an indicator of an aggressive potential. ${ }^{2}$ The up-regulation of the lipid metabolism associated pathway has been reported in many malignancies, including breast cancer, retinoblastoma, lung cancer, and colon cancer. . $^{3-6}$

Although lipid droplets (LDs) are almost ubiquitously present in eukaryotic cells, lipid storage is markedly increased in diseases associated with tissue damage or ischemia, as seen in atherosclerosis or organ infarct. ${ }^{7}$ Visualization of LDs best be facilitated by the immunohistochemical expression of adipophilin, a vehicle of small LDs in non-adipogenic cells. ${ }^{8}$ The role of adipophilin in cancer was recently investigated, and it was found to be not only a diag- nostic marker, but also an independent poor prognostic marker for certain cancers, including clear cell renal cell carcinoma and brain glioma. ${ }^{9,10}$ However, studies about the prognostic value of adipophilin in lung cancer were limited, and the association between the adipophilin and hypoxic ischemic pathways remains unclear. The objective of this study was to investigate the clinicopathologic correlation between adipophilin and hypoxic signaling molecules in primary adenocarcinoma of the lung.

\section{MATERIALS AND METHODS}

\section{Patients and tumor samples}

Cases were selected from the archives of biopsied or resected lung adenocarcinoma samples from the Seoul National University Boramae Hostpital, between June 2005 and December 2012, and from the Seoul National University Hospital between Feb- 
ruary 1996 and April 2009. Patients with primary lung adenocarcioma without a prior history of other malignancies or preoperative treatment were included in this study. A histological subtype of primary lung adenocarcinoma was reviewed and classified according to the 2015 World Health Organization classification. Tissue microarray blocks (TMAs) were constructed from the most representative tumor areas and consisted of two cores of tumor samples with diameters of $0.2 \mathrm{~cm}$. Most samples were from resected lung specimens; however, seven were from biopsy samples, which were larger than $1 \mathrm{~cm}$ in diameter. Clinical information was retrieved from electric medical records. Clinical staging was estimated according to the American Joint Committee on Cancer, eighth grading system. ${ }^{11}$ Overall survival (OS) was measured from the date of biopsy or surgery until the time of death or the last follow-up. Progression-free survival (PFS) was measured from the date of biopsy or surgery until disease progression (i.e., recurrence or metastasis) or death. This study was approved by the Institutional Review Board of Seoul National University Boramae Hospital (20180706/10-2018-69/081) and patient consent was waived.

\section{Immunohistochemical staining}

Protein expression levels of the adipophilin and hypoxic signaling markers were assessed by immunohistochemistry (IHC) from TMA blocks using an automated immunostainer (Benchmark Ventana, Tucson, AZ, USA) following the manufacturer's recommended procedure. The primary antibodies used in this study were as follows: adipophilin (1:2; Progen Biotechnik, Heidelberg, Germany), heat shock protein 27 (HSP27, 1:1,000; Novusbio, Littleton, CO, USA), carbonic anhydrase IX (CAIX, 1:1,000; Novusbio), and hypoxia-inducible factor $1 \alpha$ (HIF $1 \alpha$, 1:200, Abcam, Cambridge, UK). Expression of adipophilin was classified as positive if at least $5 \%$ of tumor cells showed cytoplasmic staining as described in a previous study. ${ }^{12}$ Other protein markers were evaluated by the $\mathrm{H}$-score: intensity multiplied by percentage of positive cells. Cases were regarded as positive when the $\mathrm{H}$-score was greater than 10 . All cases were independently reviewed by two pathologists (S.A.S and J.E.K), and agreement was reached for discordant cases.

\section{Statistical analysis}

All statistical analyses were performed using SPSS ver. 21.0 (IBM Co., Armonk, NY, USA). Correlations between the IHC results and clinicopathologic parameters were assessed with the chi-square test or Fisher exact test for nominal variables, and the Spearman's rank test for numeric variables. A univariable survival analysis was performed using Kaplan-Meier analysis with the logrank test. The Cox multiple regression model was generated to confirm independent prognostic markers in the multivariable survival analysis. Statistical significance was considered when the

Table 1. Summary of the clinicopathologic characteristics of patients

\begin{tabular}{|c|c|}
\hline Parameter & No. $(\%)(n=230)$ \\
\hline \multicolumn{2}{|l|}{ Sex } \\
\hline Male & $108(47.0)$ \\
\hline Female & $122(53.0)$ \\
\hline \multicolumn{2}{|l|}{ Age (yr) } \\
\hline$\leq 60$ & $92(40.0)$ \\
\hline$>60$ & $138(60.0)$ \\
\hline \multicolumn{2}{|l|}{ Smoking status } \\
\hline Never & 136 (59.1) \\
\hline Former/current & 88 (38.3) \\
\hline \multicolumn{2}{|c|}{ TNM category (8th) } \\
\hline I & $61(26.5)$ \\
\hline$\|$ & 97 (42.2) \\
\hline $\mid+\|$ & $158(68.7)$ \\
\hline III & 25 (10.9) \\
\hline IV & $15(6.5)$ \\
\hline$I I I+I V$ & $40(17.4)$ \\
\hline \multicolumn{2}{|l|}{ Tumor size $(\mathrm{cm})$} \\
\hline$<3$ & $103(44.8)$ \\
\hline$\geq 3$ & $92(40.0)$ \\
\hline \multicolumn{2}{|l|}{ LN metastasis } \\
\hline Negative & $125(54.3)$ \\
\hline Positive & 76 (33.0) \\
\hline \multicolumn{2}{|c|}{ Distant metastasis } \\
\hline Negative & $186(80.9)$ \\
\hline Positive & 28 (12.2) \\
\hline \multicolumn{2}{|l|}{ EGFR } \\
\hline Wild type & 77 (33.5) \\
\hline Mutated & 83 (36.1) \\
\hline Not tested & $70(30.4)$ \\
\hline \multicolumn{2}{|l|}{ KRAS } \\
\hline Wild type & $119(51.7)$ \\
\hline Mutated & $12(5.2)$ \\
\hline Not tested & $99(43.0)$ \\
\hline \multicolumn{2}{|l|}{$A L K$} \\
\hline Wild type & $225(97.8)$ \\
\hline Translocation & $5(2.2)$ \\
\hline \multicolumn{2}{|l|}{ Histologic subtype } \\
\hline Lepidic & $9(3.9)$ \\
\hline Acinar & $147(63.9)$ \\
\hline Papillary & $22(9.6)$ \\
\hline Solid & 28 (12.2) \\
\hline Micropapillary & $7(3.0)$ \\
\hline Others & $17(7.4)$ \\
\hline \multicolumn{2}{|l|}{ Progression } \\
\hline No & $82(35.7)$ \\
\hline Yes & $148(64.3)$ \\
\hline \multicolumn{2}{|l|}{ Death } \\
\hline No & $122(53.0)$ \\
\hline Yes & $108(47.0)$ \\
\hline
\end{tabular}

LN, lymph node; EGFR, epidermal growth factor receptor; $A L K$, anaplastic lymphoma kinase. 
two-tailed p-value was less than .05.

\section{RESULTS}

\section{Patients and samples}

The clinicopathologic characteristics for all of the cases are summarized in Table 1. A total of 230 cases (107 males and 123 females) with a median age of 63.4 years (range, 22.8 to 89.1 years) were enrolled in this study. The histologic type was lepidic in nine patients (4\%), acinar in 147 (64\%), papillary in $22(10 \%)$, solid in 28 (12\%), and micropapillary in seven cases (3\%). The epidermal growth factor receptor (EGFR) mutation was found in 83 of the 160 tested samples (52\%). The KRAS mutation was present in 12 of the 131 tested samples (9\%), and the anaplastic lymphoma kinase $(A L K)$ translocation was identified in five of the 230 samples. At the time of initial diagnosis, 61 patients were stage I, 97 patients were stage II, 25 patients were stage III, and 15 patients were stage IV, all according to the eighth edition of TNM classification.

\section{Immunohistochemical results}

Adipophilin expression in the cytoplasm had a spotty granular pattern, as described in the manufacturer's guidelines. Normal lung parenchymal cells were adipophilin-negative, except for alveolar macrophages, which served as the positive control. ${ }^{12}$ Overall, 30 of the 230 cases $(13 \%)$ were adipophilin-positive. Most cases (19 of 30, 63\%) showed pan-cytopalsmic patterns, while 11 (37\%) showed subnuclear basal staining patterns (Fig. 1). Positive expression of HSP27 was found in 146 cases (63\%), while CAIX showed positive staining in the cytoplasm in 129 cases (56\%). However, HIF1 $\alpha$ was only positive in two cases (1\%) (Fig. 2).

\section{Association of protein expression with clinicopathologic features}

Adipophilin positivity was significantly higher in males and in the solid histologic subtype (Fig. 3), and correlated with HSP27 expression (all p <.05). Expression of HSP27 was associated with smaller tumor sizes, low TNM stages, frequent EGFR mutation, and negative or low HIF1 $\alpha$ (all $\mathrm{p}<.01)$.
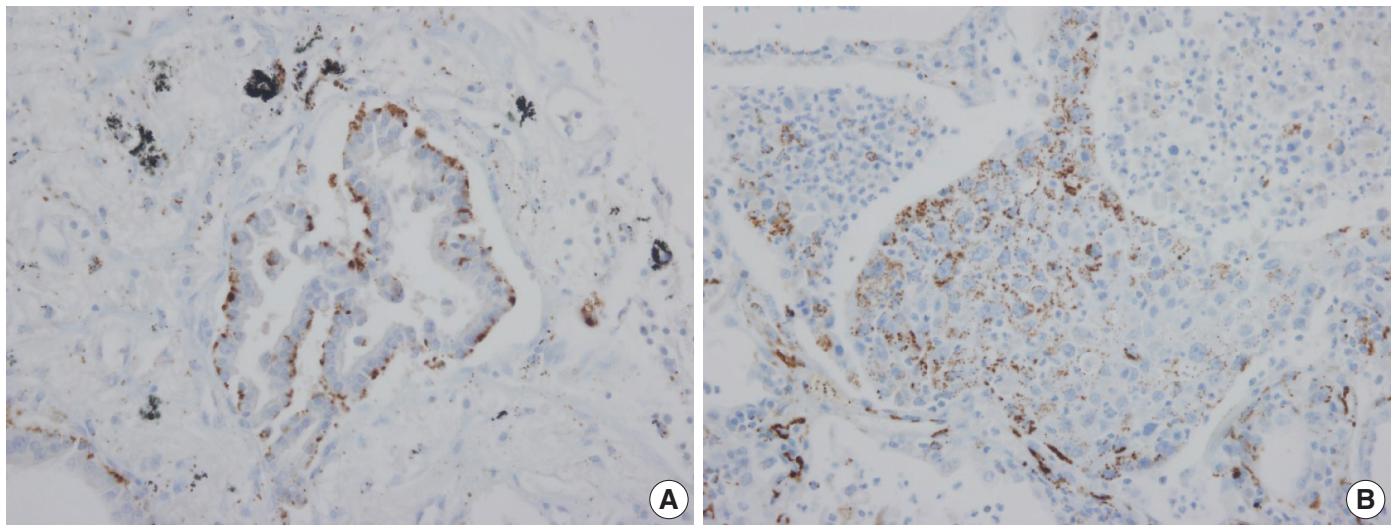

Fig. 1. Immunohistochemical staining pattern of Adipophilin in adenocarcinoma. (A) Subnuclear basal staining pattern in acinar type adenocarcinoma. (B) Pancytoplasmic pattern in solid type adenocarcinoma.
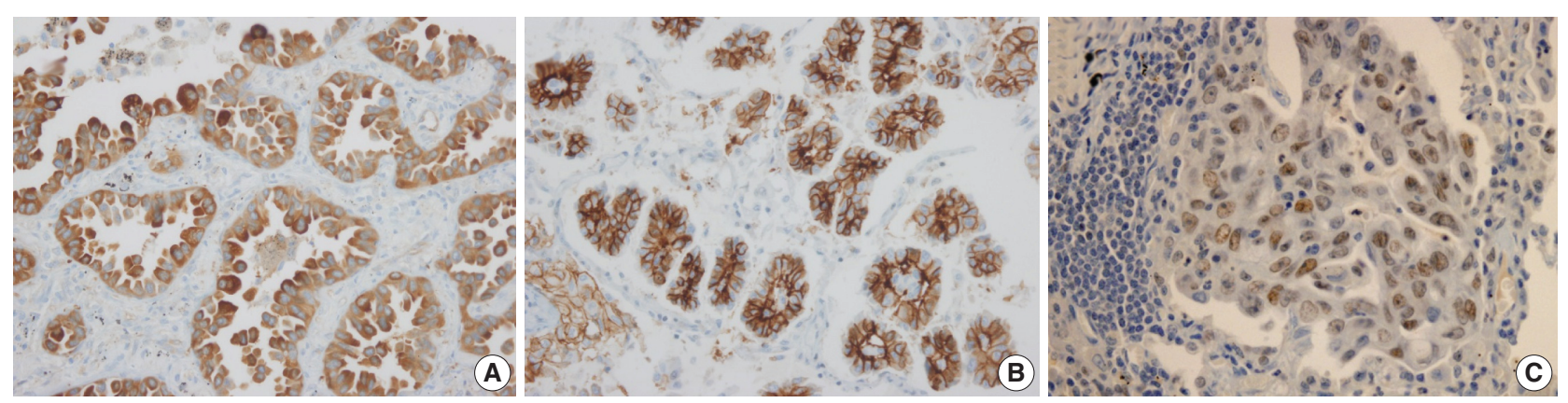

Fig. 2. Expression of hypoxic signaling proteins in lung adenocarcinoma. Strong cytoplasmic positivity of heat shock protein 27 (A) and carbonic anhydrase IX (B) is found. Nuclear expression of hypoxia-inducible factor $1 \alpha$ is only focally present (C). 


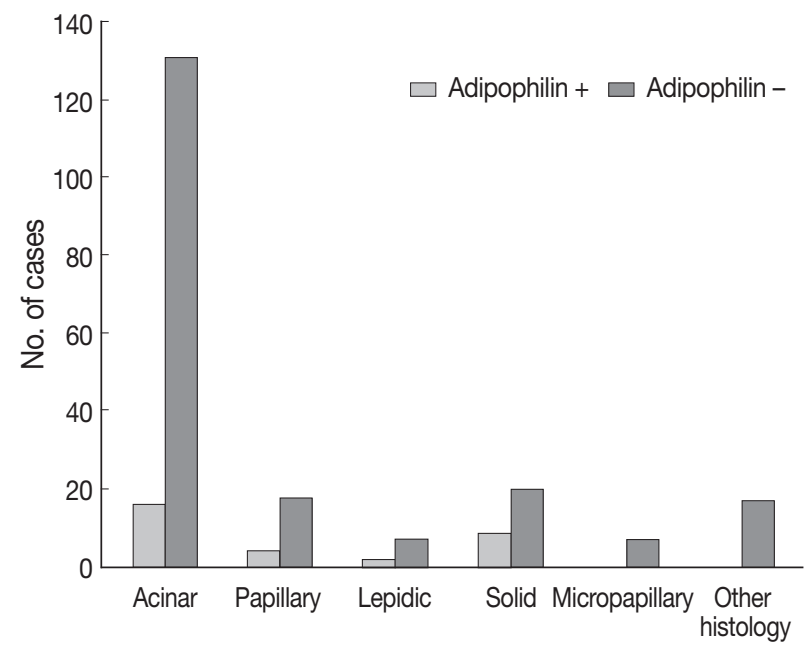

Fig. 3. Adipophilin expression according to predominant subtype of adenocarcinoma.

\section{Clinical significance of expression of adipophilin or HSP27}

The median follow-up of patients was 49.55 months (range, 1.2 to 162.7 months). Recurrence or progression was found in 148 patients, and 108 patients were deceased during the followup period. In the univariable survival analysis, patients with adipophilin positive tumors showed significantly shorter PFS (median, 17.2 months vs 18.4 months; $\mathrm{p}=.041$ ) compared to those with adipophilin negative tumors, although there was no significant difference in OS. However, the expression of HSP27 was associated with better OS and PFS ( $\mathrm{p}=.032$ and $\mathrm{p}=.002$, respectively) (Fig. 4). Other poor prognostic markers included younger patients, vascular invasion, larger tumor sizes, solid histology, and advanced TNM stages. The multivariable Cox regression analysis was performed after integrating parameters were found to have prognostic significances in the univariable analysis. As a result, adipophilin expression was an independent marker of disease progression only, whereas HSP27 expression indicated better survival in both OS and PFS (Table 2).

\section{DISCUSSION}

The goal of this study was to investigate the association of lipid metabolism with hypoxic signaling and to determine clinicopathologic significances of these molecules in primary lung adenocarcinoma. Our results showed that adipophilin was more frequently expressed in male patients with solid variant adenocarcinoma. Its expression was associated with HSP27. Moreover, the expression of adipophilin or HSP27 was an independent prognostic factor, although they behaved in a reciprocal manner.

Very few studies have focused on lipid metabolism or adi- pophilin in lung cancer, and its pathologic role and clinical significance remain unclear. One previous study reported that the adipophilin expression level was significantly higher in lung adenocarcinoma than in squamous cell carcinoma. However, that study failed to find any clinicopathologic significance, primarily due to insufficient numbers of cohorts. ${ }^{13}$ In contrast, the positive rate of adipophilin expression in squamous cell carcinoma in our study was higher than that in adenocarcinoma (25\% vs $13 \%$, unpublished data). Another recent study by Fujimoto et al. ${ }^{12}$ found that adipophilin positivity was associated with apocrinelike features and worse outcomes in lung adenocarcinoma. Our results partially coincided with that study with regard to prognostication. However, we could not find apocrine-like histologic patterns represented by the eosinophilic granular cytoplasm. The only relative predominance of a solid histologic pattern in adipophilin positive tumors was found in our study. Among various malignancies, one of the best-known examples of adipophilin positive tumors are renal cell carcinomas and ductal carcinomas of the breast, characterized by frequent solid architectures and plump cytoplasms. ${ }^{911}$ Taken together, these results suggested that the lipidogenic pathway is activated in adenocarcinoma with solid phenotypes in various organs.

The accumulation of $\mathrm{LDs}$ is a common finding in the tissues after ischemic injury, as seen in atherosclerosis or organ infarct.' Ischemia followed by activation of hypoxic signaling is a typical finding of malignant tumors, and is strongly associated with aggressive behavior. ${ }^{910}$ Recent studies demonstrated that the fractional contribution of glutamine to fatty acid synthesis increased during hypoxia. ${ }^{15}$ We hypothesized that the coordination of metabolic deregulation and hypoxic signaling contributed to the biologic aggressiveness of lung adenocarcinomas. The strong correlation between adipophilin expression and HSP27 in our study supported this hypothesis. To further verify our hypothesis, we examined several well-known biomarkers in hypoxic signaling, including HIF1 $\alpha$, a master regulator of hypoxia and related proteins. Although the expression of HSP27 and CAIX was frequently found regardless of histologic type, the expression of HIF1 $\alpha$ was particularly low. One possible cause of such low positivity of HIF1 $\alpha$ might be the extremely short half-life of that protein. The ubiquitin-proteasome pathway is responsible for the stability of HIF1 $\alpha$, which is rapidly degraded in normoxia, resulting in undetectable levels in immunohistochemistries. ${ }^{16}$ Previous studies found inconsistent results of HIF1 $\alpha$ expression in various cancers, and many studies suggested that HIF1 $\alpha$ stability was regulated in a cell-type specific manner. ${ }^{16}$ Although we cannot completely explain the low HIF1 $\alpha$ incidence in our subjects, many of the 

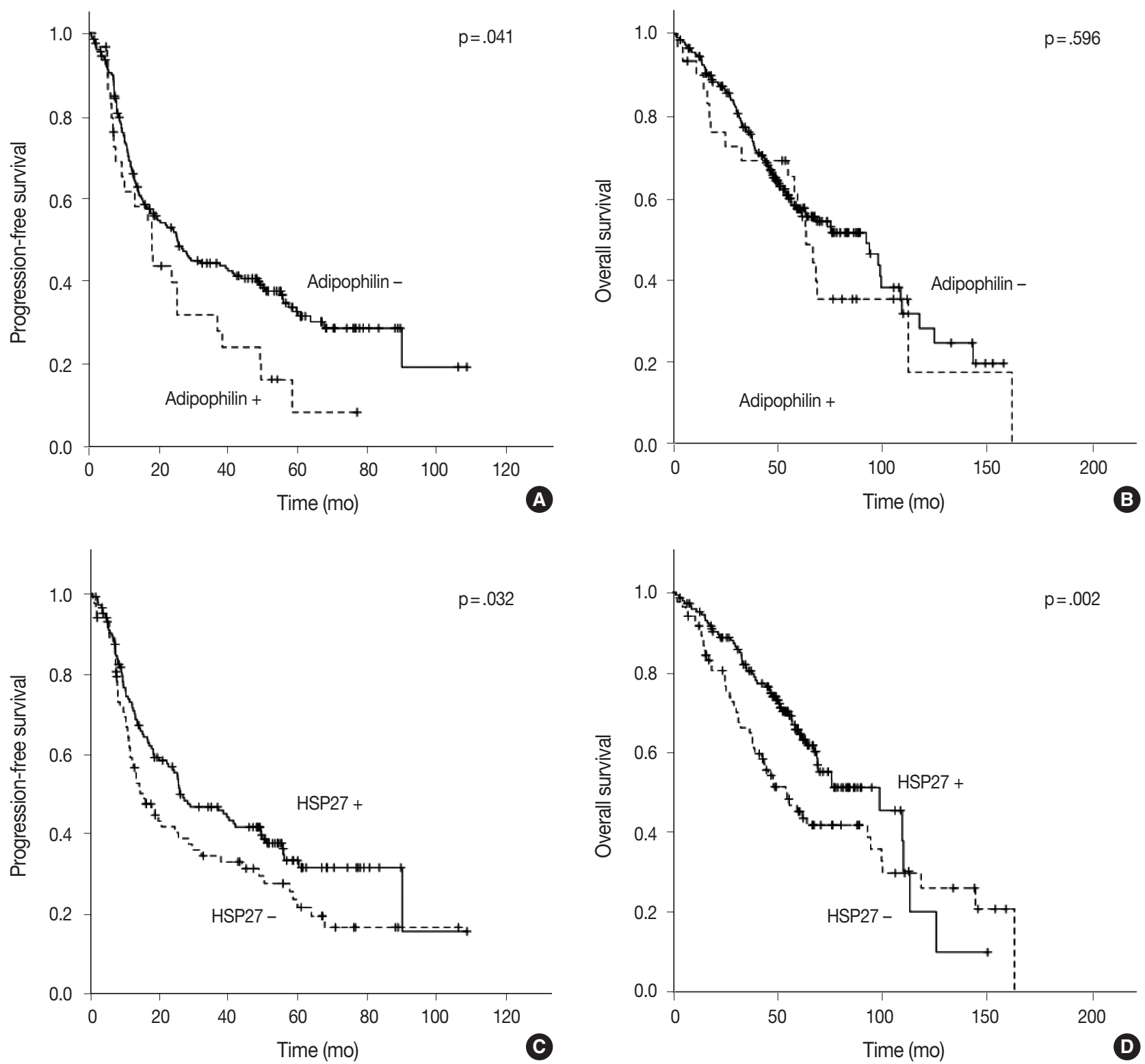

Fig. 4. Results of univariable survival analysis represented by Kaplan-Meier plots according to protein expression. Expression of adipophilin is significantly associated with worse progression-free survival (PFS) (A) but is not related to overall survival (OS) (B). Heat shock protein 27 shows poor prognostic impacts in both PFS (C) and OS (D).

Table 2. Results of multivariate Cox regression analysis

\begin{tabular}{|c|c|c|c|c|c|c|}
\hline \multirow{2}{*}{ Variable } & \multicolumn{3}{|c|}{ Overall survival } & \multicolumn{3}{|c|}{ Progression free survival } \\
\hline & $\mathrm{HR}$ & $95 \% \mathrm{Cl}$ & $p$-value & $\mathrm{HR}$ & $95 \% \mathrm{Cl}$ & $p$-value \\
\hline Age (>60 yr) & 0.542 & $0.250-1.173$ & .120 & 0.561 & $0.260-1.208$ & .140 \\
\hline Tumor size ( $\geq 3 \mathrm{~cm})$ & 1.168 & $0.511-2.668$ & .713 & 1.627 & $0.686-3.858$ & .269 \\
\hline TNM (III, IV vs I, II) & 8.355 & $3.486-20.029$ & $<.001^{\star \star *}$ & 8.318 & 3.322-20.832 & $<.001^{* * *}$ \\
\hline EGFR mutation & 0.637 & $0.270-1.501$ & .302 & 1.209 & $0.531-2.751$ & .651 \\
\hline Vascular invasion & 1.132 & $0.456-2.808$ & .789 & 1.024 & $0.415-2.524$ & .959 \\
\hline Adipophilin ( $\geq 5 \%)$ & 4.467 & $1.783-11.190$ & $.001^{* *}$ & 4.476 & $1.792-11.183$ & $.001^{* *}$ \\
\hline HSP27 ( $\geq 0$ by H score) & 0.280 & $0.123-0.641$ & $.003^{* *}$ & 0.391 & $0.170-0.897$ & $.027^{*}$ \\
\hline
\end{tabular}

$\mathrm{HR}$, hazard ratio; $\mathrm{Cl}$, confidence interval; EGFR, epidermal growth factor receptor; HSP27, heat shock protein 27.

Statistically significant ${ }^{*} \mathrm{p}<.05,{ }^{* *} \mathrm{p}<.01,{ }^{* * *} \mathrm{p}<.001$. 
factors listed above might have contributed to its low immunoreactivity.

An interesting observation of this study was the reciprocal action of adipophilin and HSP27 in terms of prognostication. As expected, adipophilin was an independent negative prognostic factor of lung adenocarcinoma. However, its prediction was inversed in HSP27, even with the strong correlation between two proteins. It is believed that HSP27 has either a protective or a counter-protective role in various malignancies. ${ }^{17}$ Typically, HSP27 responds to hypoxic conditions to lower reactive oxygen species in a protective way. This protection also provides a shelter for both normal and cancer cells. In this study, the frequent expression of HSP27 or CAIX indicated activation of hypoxic signaling and possible link of metabolic deregulation. However, further research, including functional studies, are needed to better understand the precise interaction between these proteins.

In conclusion, adipophilin positivity was common in solid histologic types of lung adenocarcinoma, and was associated with adverse outcomes. The deregulation of lipid metabolism with hypoxic signaling might play a role in the pathogenesis of lung adenocarcinoma.

\section{ORCID}

Ji Eun Kim: https://orcid.org/0000-0002-2156-9875

\section{Conflicts of Interest}

No potential conflict of interest relevant to this article was reported.

\section{Acknowledgments}

This study was supported by the Grant of National Research Foundation of Korea (2017R1A2B4005052).

\section{REFERENCES}

1. Hsu PP, Sabatini DM. Cancer cell metabolism: Warburg and beyond. Cell 2008; 134: 703-7.

2. Beloribi-Djefaflia S, Vasseur S, Guillaumond F. Lipid metabolic reprogramming in cancer cells. Oncogenesis 2016; 5: e189.

3. Alo PL, Visca P, Marci A, Mangoni A, Botti C, Di Tondo U. Expression of fatty acid synthase (FAS) as a predictor of recurrence in stage I breast carcinoma patients. Cancer 1996; 77: 474-82.

4. Camassei FD, Cozza R, Acquaviva A, et al. Expression of the lipogenic enzyme fatty acid synthase (FAS) in retinoblastoma and its correlation with tumor aggressiveness. Invest Ophthalmol Vis Sci 2003; 44: 2399-403.

5. Migita T, Narita T, Nomura K, et al. ATP citrate lyase: activation and therapeutic implications in non-small cell lung cancer. Cancer Res 2008; 68: 8547-54.

6. Luque-García JL, Martínez-Torrecuadrada JL, Epifano C, Cañamero M, Babel I, Casal JI. Differential protein expression on the cell surface of colorectal cancer cells associated to tumor metastasis. Proteomics 2010; 10: 940-52.

7. Straub BK, Gyoengyoesi B, Koenig M, et al. Adipophilin/perilipin-2 as a lipid droplet-specific marker for metabolically active cells and diseases associated with metabolic dysregulation. Histopathology 2013; 62: 617-31.

8. Heid HW, Moll R, Schwetlick I, Rackwitz HR, Keenan TW. Adipophilin is a specific marker of lipid accumulation in diverse cell types and diseases. Cell Tissue Res 1998; 294: 309-21.

9. Tolkach Y, Luders C, Meller S, Jung K, Stephan C, Kristiansen G. Adipophilin as prognostic biomarker in clear cell renal cell carcinoma. Oncotarget 2017; 8: 28672-82.

10. Kohe S, Colmenero I, McConville C, Peet A. Immunohistochemical staining of lipid droplets with adipophilin in paraffin-embedded glioma tissue identifies an association between lipid droplets and tumour grade. J Histol Histopathol 2017; 4: 4.

11. Brierley JD, Gospodarowicz MK, Wittekind C. TNM classfication of malignant tumours. 8th ed. Oxford: Wiley-Blackwell, 2017.

12. Fujimoto M, Yoshizawa A, Sumiyoshi S, et al. Adipophilin expression in lung adenocarcinoma is associated with apocrine-like features and poor clinical prognosis: an immunohistochemical study of 328 cases. Histopathology 2017; 70: 232-41.

13. Zhang XD, Li W, Zhang N, et al. Identification of adipophilin as a potential diagnostic tumor marker for lung adenocarcinoma. Int J Clin Exp Med 2014; 7: 1190-6.

14. Moritani S, Ichihara S, Hasegawa M, et al. Intracytoplasmic lipid accumulation in apocrine carcinoma of the breast evaluated with adipophilin immunoreactivity: a possible link between apocrine carcinoma and lipid-rich carcinoma. Am J Surg Pathol 2011; 35: 861-7.

15. Kamphorst JJ, Cross JR, Fan J, et al. Hypoxic and Ras-transformed cells support growth by scavenging unsaturated fatty acids from lysophospholipids. Proc Natl Acad Sci U S A 2013; 110: 8882-7.

16. Zheng X, Ruas JL, Cao R, et al. Cell-type-specific regulation of degradation of hypoxia-inducible factor 1 alpha: role of subcellular compartmentalization. Mol Cell Biol 2006; 26: 4628-41.

17. Kaigorodova EV, Bogatyuk MV. Heat shock proteins as prognostic markers of cancer. Curr Cancer Drug Targets 2014; 14: 713-26. 